Tasneem Atef Dalgamouni, Shatha Al-Khanji, Maroua Cherif, Rihab Rasheed, Touria Bounnit, Dr. Hareb Aljabri, Dr. Imen Saadaoui, Dr. Radhouane Ben Hamadou.

\title{
Isolation, cultivation, and characterization of novel local marine micro-algae for aquaculture feed supplement production
}

\section{ABSTRACT}

Aquaculture is considered as a promising alternative to support the food demands of the ever-increasing population. Currently, this sector faces several challenges such as using fishmeal, which is unsustainable and expensive. Therefore, it is necessary to identify an alternative feed component that is sustainable, cost-effective and can provide the essential nutrients required by the fish. In this context, microalgae are considered as a viable source of proteins, lipids, polysaccharides and high-value products (HVPs) such as essential fatty acids, amino acids and vitamins. They play a vital role in the marine food chain and hence can be easily assimilated by the fish. The current research targeted the isolation, identification and characterization of novel marine microalgae from Qatar coastline to produce aquaculture feed supplement.

For that, environmental samples have been subjected for microalgae isolation. The isolated microorganisms belong to mainly three classes: diatoms, cyanobacteria and green algae. Among them, three have been well characterized in terms of growth rate and metabolites profiling.

\section{METHODOLOGY}

\section{Sample isolation \& morphological characterization}

Samples were isolated and purified using common plating method $F / 2$ and $B G 11$ mediums.

Light microscopy is used to determine the morphological characteristics.

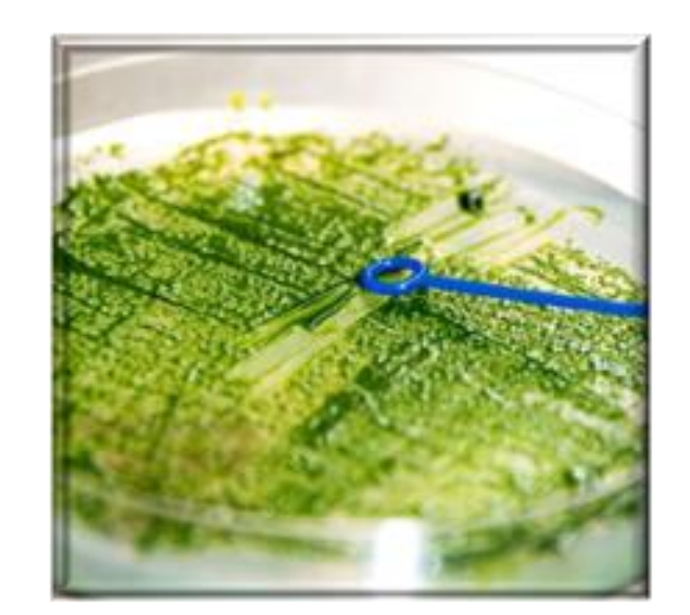

\section{Molecular identification}

The Genomic DNA was extracted and PCR amplification was performed using the genomic markers (18SrDNA, 16S rDNA). The PCR products where purified then sequenced and bioinformatics analysis was done

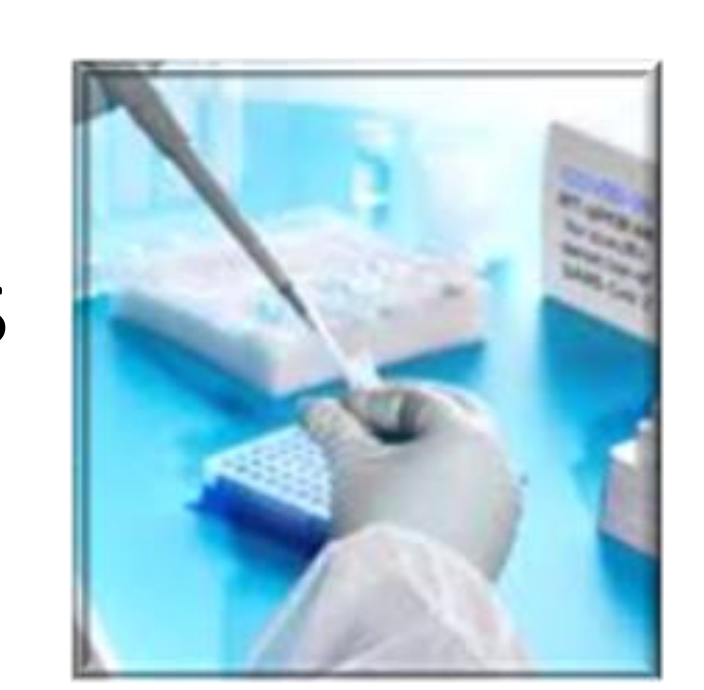

\section{Growth rate studies}

Optical Density was measured daily using spectrophotometry at $750 \mathrm{~nm}$.

Growth rate $(u)$ is: $\mu=\ln X 2-\ln X 1 / \mathrm{t} 2-\mathrm{t} 1$ where $X 1$ and $X 2$ are optic densities at times $\mathrm{t} 1$ and $\mathrm{t} 2$.

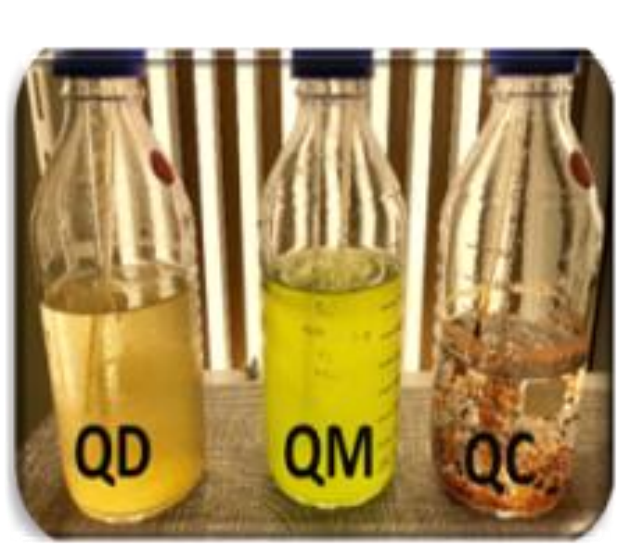

\section{Cell dry-weight determination}

$10-\mathrm{ml}$ of the culture was filtered through $0.47 \mathrm{~mm}$ dry weight filters and kept at $80^{\circ} \mathrm{C}$ for $24 \mathrm{~h}$, then transferred to desiccators for dehydration and dry weight calculations

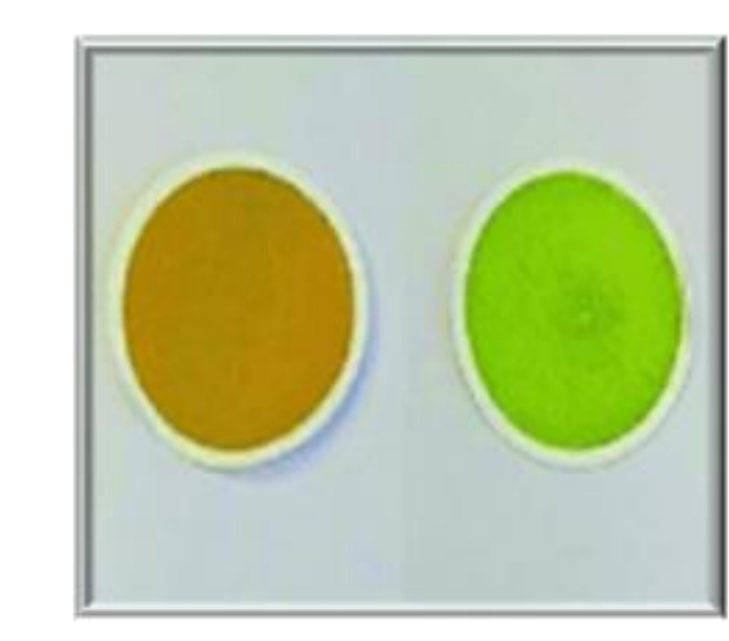

5. Protein and lipid extraction Lowry-BSA method is used to determine the protein content (\%).

Total lipids was extracted using Folch Method.

The total lipid content will be determined gravimetrically.

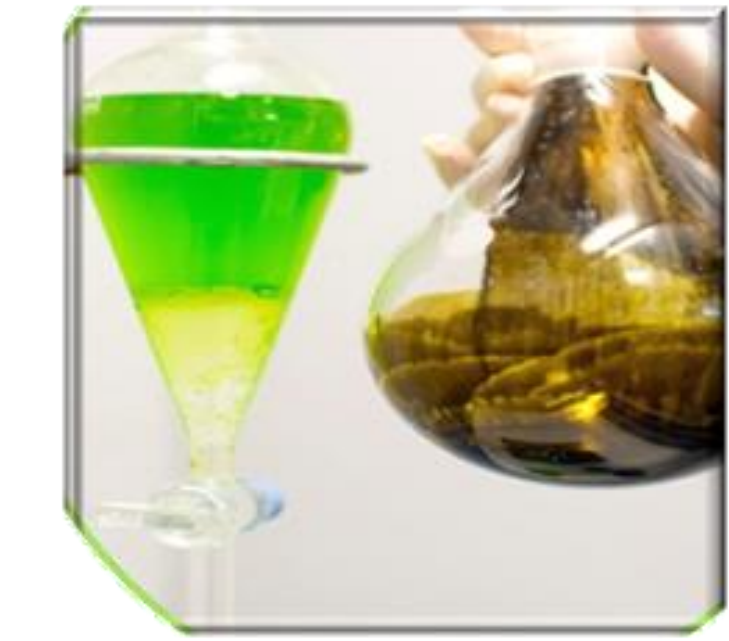

6. Crude extraction of algae carotenoid Carotenoids is extracted via maceration using methanol.

Spectrophotometry was used at $480 \mathrm{~nm}$ to determine the carotenoid concentration.

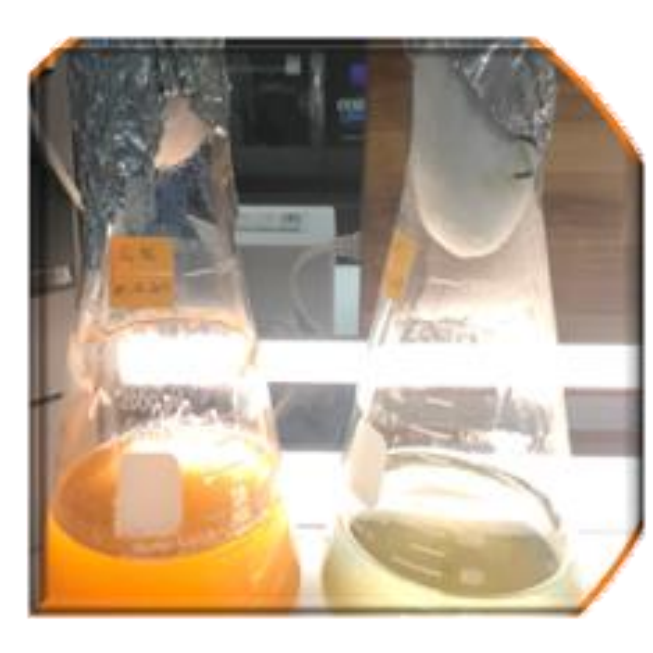

\section{CONCLUSION}

Based on the metabolites and he growth analysis, a rich feed supplement blending the three isolates can be considered as an alternative to fishmeal. well balanced and with high nutritional value.

\section{REFERENCES}

\section{OBJECTIVES}

- Isolation, Identification and Characterization of novel marine microalgae to produce aquaculture feed supplement.

- Cultivation and assessment of the growth parameters and the metabolites content.

\section{LITERATURE REVIEW}

Global total capture fisheries production reached 91 million tons in 2016, as per FAO's recent statistics. However, such production didn't meet the worldwide demand due to the drastic population increase. For these reasons, aquaculture becomes of particular interest worldwide as a viable alternative to produce a large quantity of seafood (Patrik et al., 2017). Marine microalgae are considered a very promising alternative for aqua feed production since they represent the main primary food producers for the aquatic food chain (Borowitzka 1998).

\section{RESULTS and DISCUSSION}

1. Isolation, purification and characterization of local marine microalgae isolates
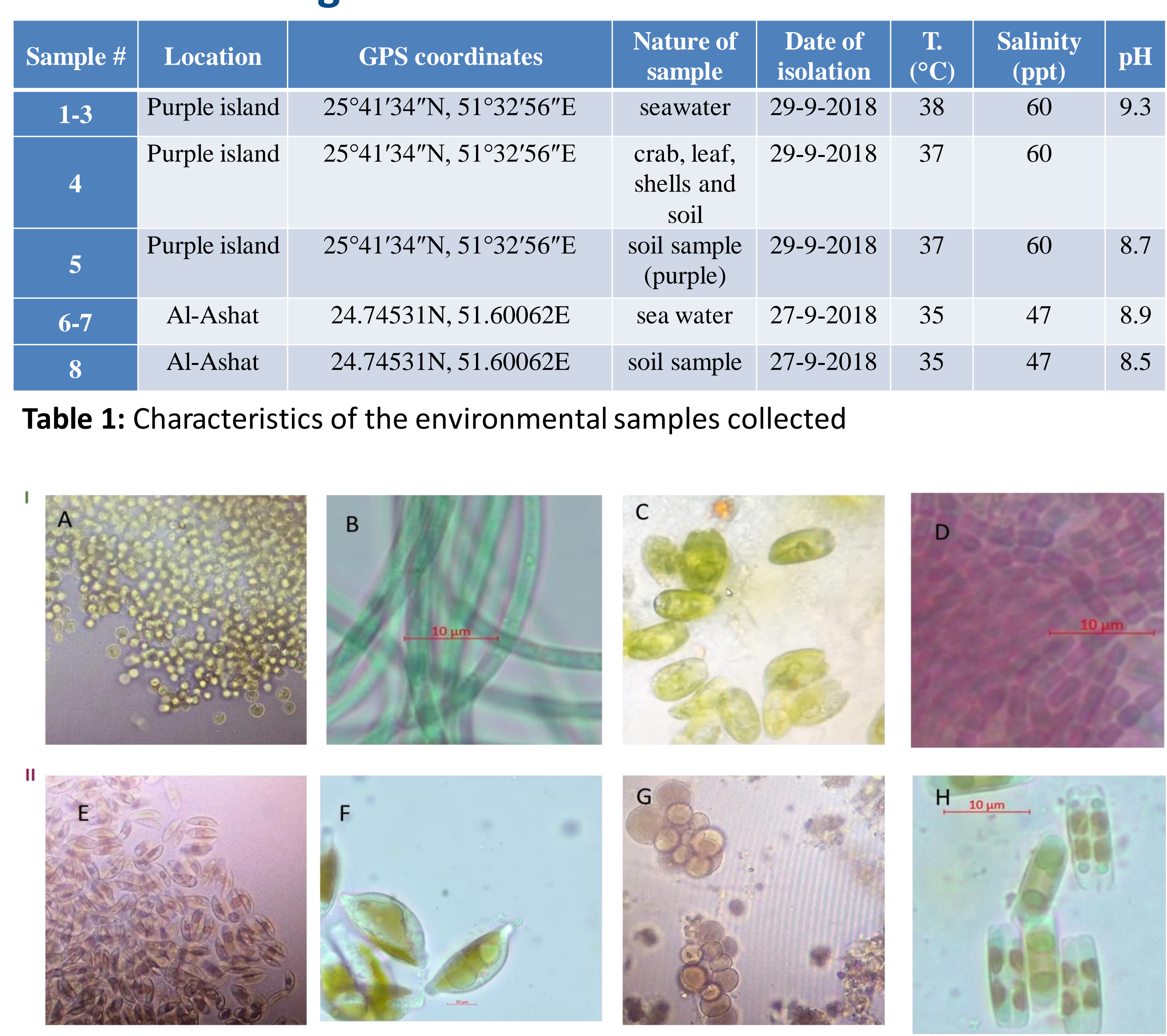

Figure 1: isolated strains

20 strains were isolated, The main novel isolated strains were Diatoms, cyanobacteria and green microalgae. They were purified and characterized morphologically using light microscopy.

$>$ It is noted that samples collected from the purple island contain mainly diatoms and purple cyanobacteria. Accordingly, these two microorganisms might be at the origin of purple color of the soil.

2. Molecular identification of the new isolates

DNA Extraction.

PCR amplification of the genomic markers, such as $16 \mathrm{~s}$ rDNA and the 18s rDNA for Microalgae and Cyanobacteria.

- PCR Product Purification to get Pure DNA

Sequencing \& bioinformatic analysis.

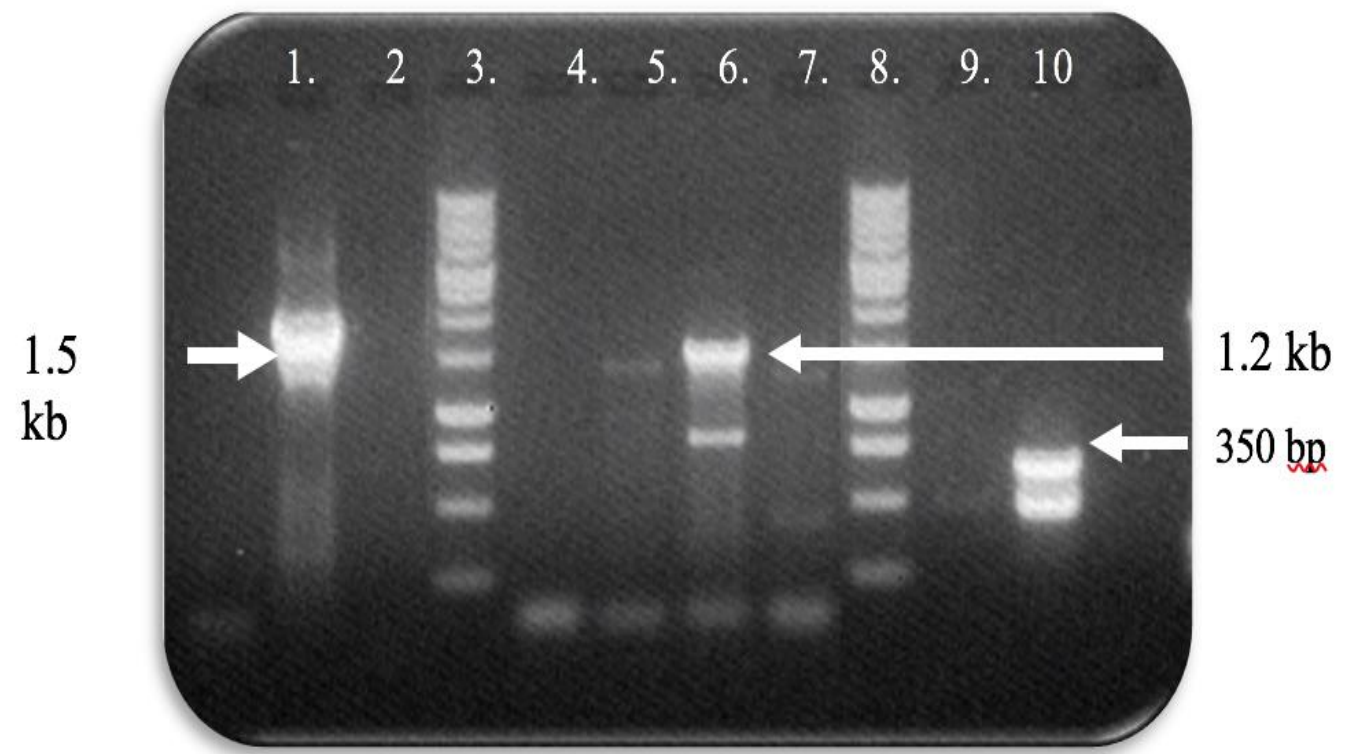

Figure2: PCR amplification of the cellected genomic markers,

The multiple alignment of the $\mathrm{OM}$ sequence proved that this strain is Tetraselmis subcoliformis (Figure 2)
3. Assessment of the Growth rate and biomass productivity

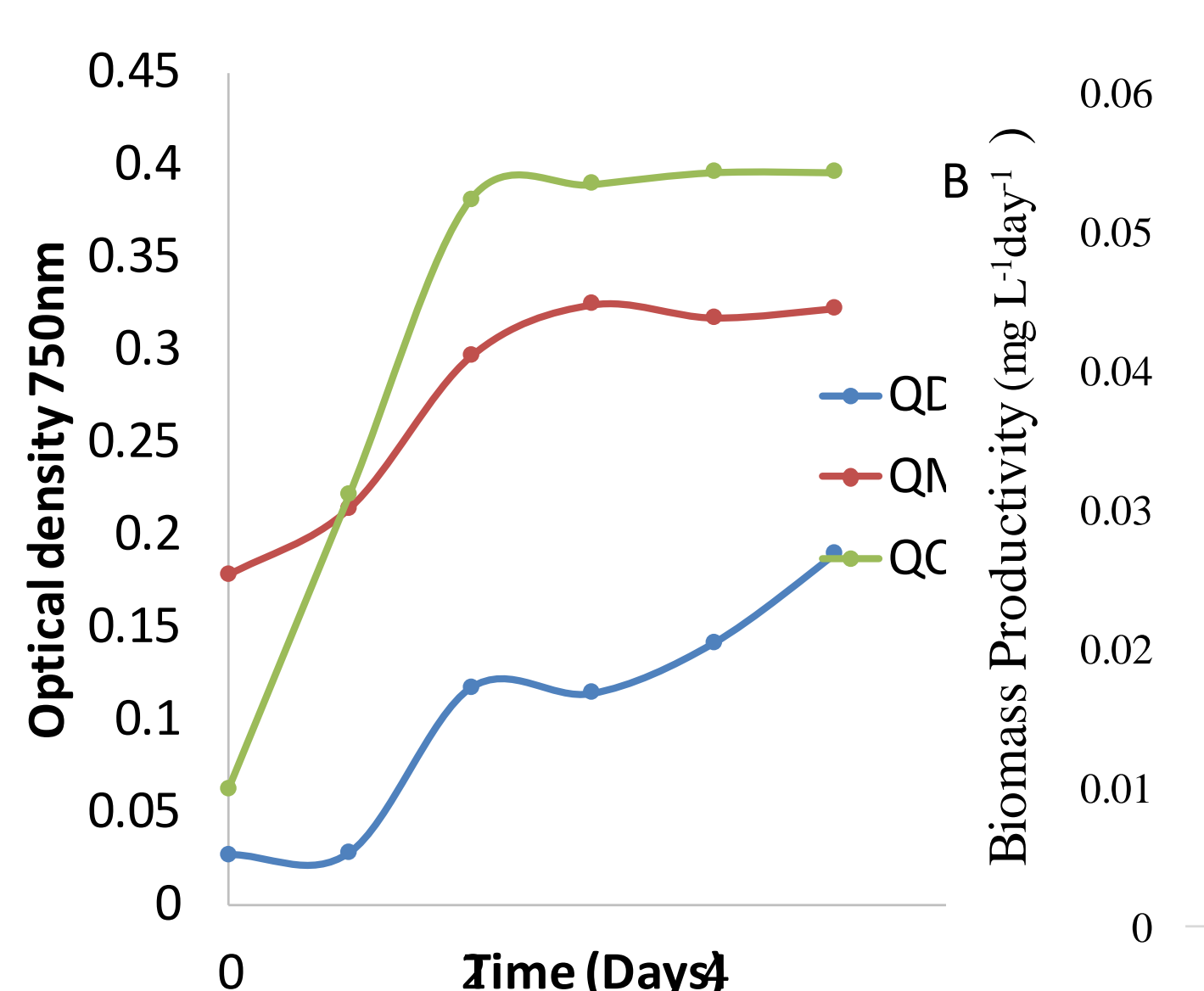

QC presents the fastest growth and highest biomass productivity of (51.4 mgL-1day-1), followed by the Tetraselmis subcoliformis. (38.2 mgL-1day-1),

4. Metabolites profiling of the three newly isolated microalgae

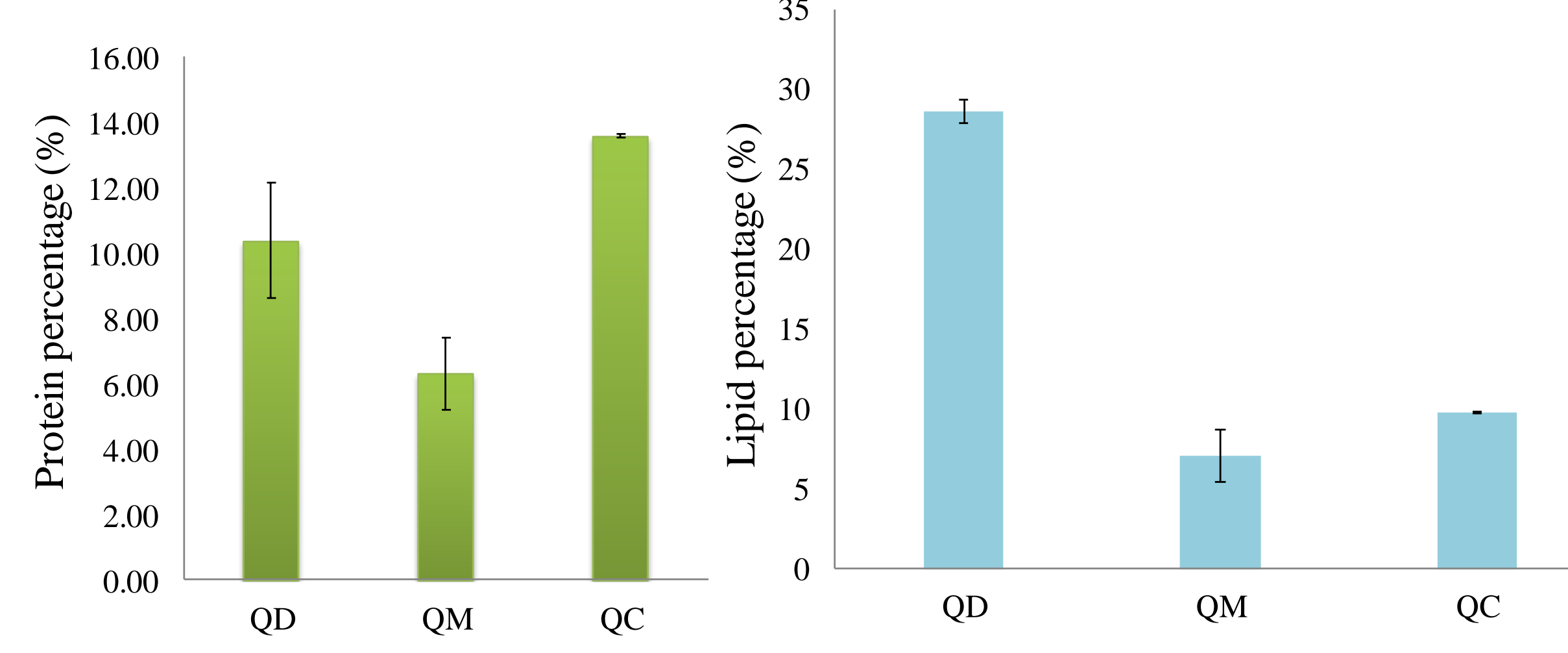

D The cyanobacteria strain (OC) presents the highest amount of protein $13.57 \% / \mathrm{g}$ dry weight.

$>$ the Diatom (QD) was the highest in lipid concentration (28.62\%).

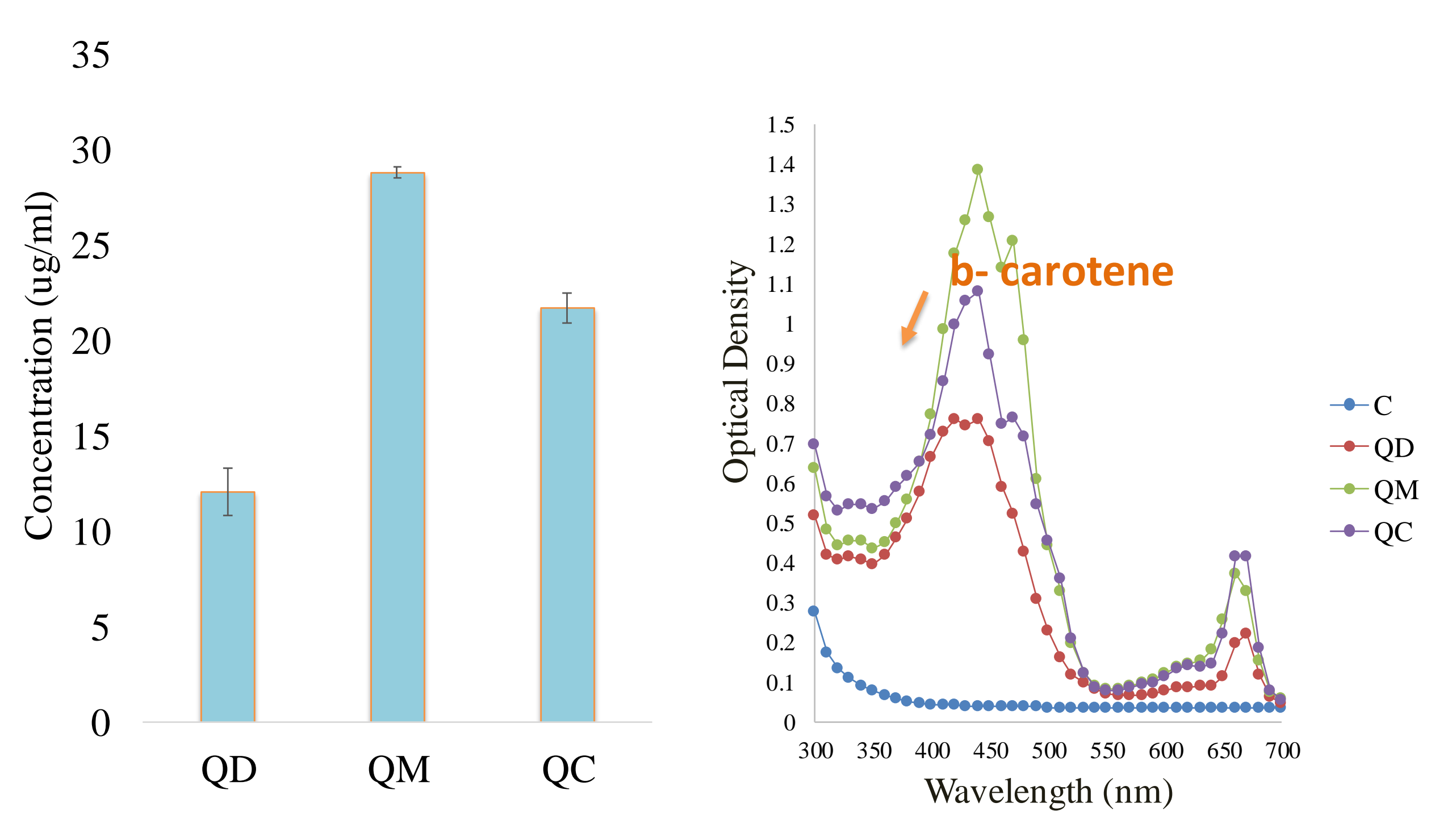

$>\mathrm{QM}$ (Tetraselmis subcoliformis) present the highest amount of carotenoids.

The deep investigation of the nature of the carotenoids using spectral scan proved the presence of $b$ - carotene.

QM represents a very good candidate for producing high quality aquafeed enriched with high value added compounds. 